\title{
High-Speed and Wide-Field Photometry with TORTORA
}

\author{
G. Greco, ${ }^{1}$ G. Beskin, ${ }^{2}$ S. Karpov, ${ }^{2}$ S. Bondar, ${ }^{3}$ C. Bartolini, ${ }^{1}$ A. Guarnieri, ${ }^{1}$ and A. Piccioni ${ }^{1}$ \\ ${ }^{1}$ Astronomy Department, Bologna University, 40126 Bologna, Italy \\ ${ }^{2}$ Special Astrophysical Observatory of Russian Academy of Science, 369167 Nizhnij Arkhyz, Russia \\ ${ }^{3}$ Institute for Precise Instrumentation, 369167 Nizhnij Arkhyz, Russia \\ Correspondence should be addressed to G. Greco, giuseppe.greco2@studio.unibo.it
}

Received 8 July 2009; Accepted 24 September 2009

Academic Editor: Alberto J. Castro-Tirado

Copyright ( $) 2010$ G. Greco et al. This is an open access article distributed under the Creative Commons Attribution License, which permits unrestricted use, distribution, and reproduction in any medium, provided the original work is properly cited.

We present the photometric analysis of the extended sky fields observed by the TORTORA optical monitoring system. The technology involved in the TORTORA camera is based on the use of a fast TV-CCD matrix with an image intensifier. This approach can both significantly reduce the readout noise and shorten the focal length following to monitor relatively large sky regions with high temporal resolution and adequate detection limit. The performance of the system has been tested using the relative magnitudes of standard stars by means of long image sequences collected at different airmasses and at various intensities of the moon illumination. As expected from the previous laboratory measurements, artifact sources are negligible and do not affect the photometric results. The following analysis is based on a large sample of images acquired by the TORTORA instrument since July 2006.

\section{Introduction}

The complete randomness of the angular distribution of the $\gamma$-ray bursts (GRBs) in the sky, along with their typical short duration, suggested us to monitor large sky regions with high temporal resolution in order to increase the probability to catch optical emissions simultaneous with the $\gamma$-ray prompt events. To perform such a task, we have proposed different search strategies [1-6] and the high-speed and wide-field FAVOR [7] and TORTORA cameras represent the more recent and promising developments. Presently TORTORA (Telescopio Ottimizzato per la Ricerca dei Transienti Ottici RApidi) is mounted on top of the REM robotic telescope at La-Silla Observatory in Chile and has been operating since May 2006 [8].

\section{Short Technical Description}

The camera consists of a main objective, an image intensifier used to down-scale and amplify the image, a transmission optics, and a fast low-noise TV-CCD matrix based on the Sony ICX285AL chip. The TV-CCD matrix is an interlinetransfer CCD with microlens raster to compensate the loss of surface area. It can operate at 7.5 frames per seconds with negligible gaps between consecutive exposures. This frametransfer regime is typical for CCDs and does not bring any significant artifacts in the image. The main objective and the transmission optics focusing unit are controlled through the PC parallel port interface. In Table 1 are reported the main technical parameters of the TORTORA camera.

The resulting data flow rate is about $20 \mathrm{MB} / \mathrm{s}$. The raw data are stored on a RAID array until the next observational night and are processed in real time by a dedicated software for detection and classification of optical transient events [9].

The difference between an ordinary imaging with a CCD camera and fast imaging with a CCD equipped with an image intensifier tube is very significant [10]. The intensifier converts each photon, detected by the photocathode, into a luminous spot on the phosphor screen that preserves the $(x, y)$ location of the event. This approach produces an efficient amplification of the intensity of the image afterwards recorded by the CCD. High gain of the image intensifier, which may be expressed as the number of resulting CCD counts per photon on the photocathode leads to effective reduction of the readout noise down to a subelectron values. Furthermore, the image intensifier is able to down-scale the image reducing the focal length and consequently increasing the field of view for a fixed CCD size. The gain of the image 
TABLE 1: Main parameters of the TORTORA camera.

\begin{tabular}{lccccc}
\hline \multicolumn{2}{c}{ Main objective } & \multicolumn{2}{c}{ Intensifier } & & CCD \\
\hline Diameter & $120 \mathrm{~mm}$ & Photocathode & S20 & Dimensions & $1388 \times 1036$ pix \\
Focal length & $150 \mathrm{~mm}$ & Diameter & $90 \mathrm{~mm}$ & Pixel scale & $81^{\prime \prime} /$ pix \\
Focal ratio & $1 / 1.2$ & Gain & 150 & Exposures & $0.13 \mathrm{~s}$ \\
Field of view & $24^{\circ} \times 32^{\circ}$ & Scaling factor & 5.5 & Pixel size & $6.5 \mathrm{micron}$ \\
& & Quantum efficiency & $10 \%$ & Readout noise & $6 \mathrm{e}^{-} / \mathrm{pix}$ \\
\hline
\end{tabular}

intensifier tube employed in TORTORA is 150 , and the focal length is reduced from $150 \mathrm{~mm}$ down to $27 \mathrm{~mm}$. However, the drawback of image intensifier is the decrease of the system quantum efficiency (Section 4.1) to a value defined by the characteristics of the photocathode, which is only $10 \%$ for an S20 photocathode used here.

\section{Data Reduction}

The real-time data processing pipeline is based on a fast differential imaging algorithm described in [10]. More detailed time series analysis may be performed a posteriori studying the images stored on the RAID array.

3.1. Preliminary Analysis. The first step of the postprocessing analysis is the dark current noise subtraction and flatfielding to compensate vignetting due to the objective design. The low-noise TV-CCD matrix employed in TORTORA operates at room temperature. The dark current component is originated from thermally generated charge in the photocathode before the amplification stage and then it will be also amplified (the dark current in image intensifiers was traditionally called the Effective Background Illumination (normally abbreviated to EBI)). The dark current is generally not an issue if short time exposures are planned. Practically thermal noise has a negligible effect on the signal-to-noise ratio of the frames acquired by TORTORA, therefore no cooling is applied to the CCD camera.

The next step is the measure of the CCD frames taking into account the distribution of the background level and the statistics of photoelectrons.

\subsection{Background Estimate. Faint stars at the limit of detec-} tion, photons of scattered light by the environment, satellites trails, cosmic-rays, or bad pixels can produce random fluctuations in a single frame. The variable background brightness is initially treated identifying pixels which are significantly higher than the surrounding one. The pixels deviating by a specified statistical amount are replaced by the median value. The nonuniformity of the frame background was reduced by approximately $15 \%$ in accordance with external conditions. This procedure is done on the whole frame, subsequently the background signal is determined within the circular shell of inner radius annulus and outer radius annulus surrounding the object without including other astronomical objects within this shell. The measured standard deviation in the stack of images is higher than that expected from pure

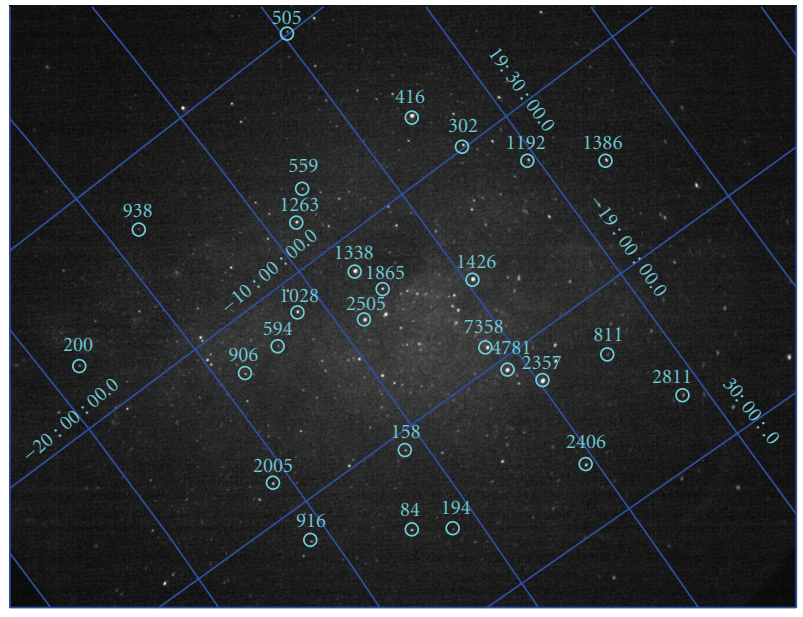

FIgURE 1: Image acquired by the TORTORA camera with an exposure time of 0.13 seconds. The selected stars are labeled using the identification number (id number) as tabulated in Tycho-2 catalogue. They have been chosen as reference stars to test the linearity and the homogeneity of the instrument (see text for more details). The center coordinates of the image are at the position: $\mathrm{RA}(\mathrm{J} 2000)=18 \mathrm{~h}: 37 \mathrm{~m}: 05.28 \mathrm{~s}, \operatorname{DEC}(\mathrm{J} 2000)=-06 \mathrm{~d}: 29^{\prime}:$ $38.04^{\prime \prime}$. Real time images of the TORTORA system are available using the public graphical user interface at the following address: http://polaris.merate.mi.astro.it/trem/.

Poisson noise especially at large count rates. Although the image intensifier overcomes the problem of readout noise, images acquired with an image intensifier suffer from gain noise. Intensifier noise is mainly caused by the discrete nature of the electron amplification process. Each photon entering the image intensifier will induce a large number of photons leaving the phosphorus screen. The variation in the gain for each incoming photon and the correlation in time of the output photons are the source of the noise. A single input photon yields a burst of output photons. The departure times of these output photons are strongly correlated in time. As a consequence, the Poisson process at the input of the intensifier yields a non-Poisson process at the output, which has an increased variance compared to ordinary Poissondistributed noise. The number of photoelectrons generated at the photocathode depends on the binomial statistics:

$$
P(r)=\left(\begin{array}{l}
N \\
r
\end{array}\right) p^{r}(1-p)^{(N-r)} .
$$




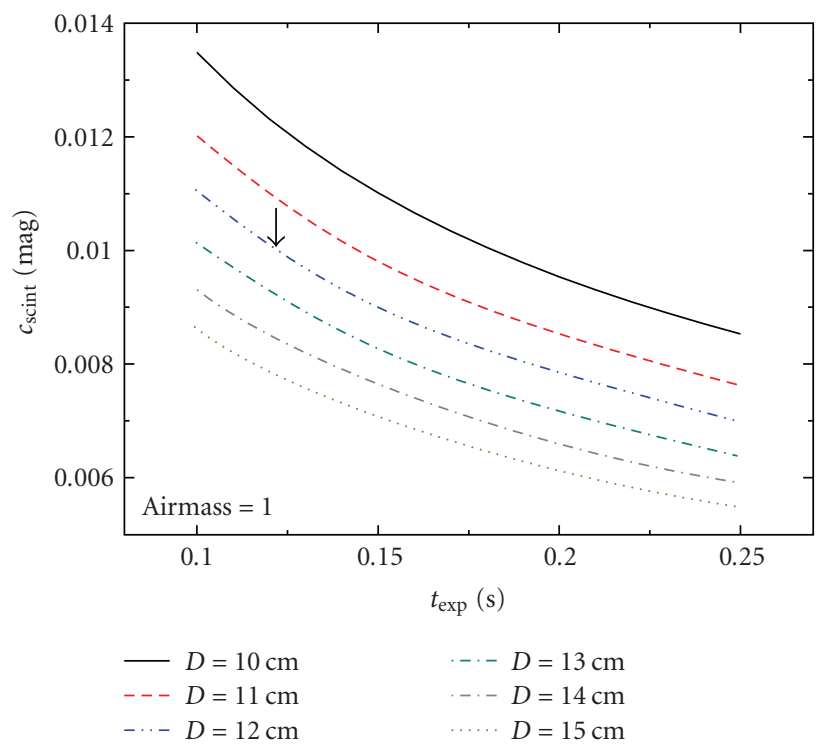

(a)

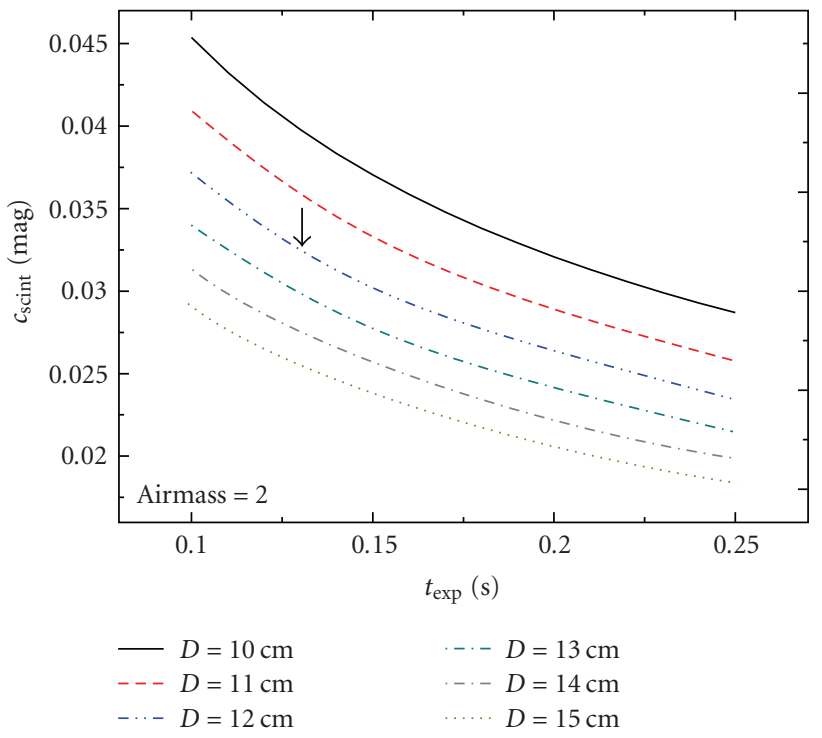

(b)

Figure 2: $c_{\text {scint }}$ versus short exposure times $t_{\text {exp }}<0.25$ seconds for different size of telescopes $(10 \mathrm{~cm} \leq D \leq 15 \mathrm{~cm})$ at a fixed values of airmass: $X=1$ (a) and $X=2$ (b). The arrows indicate the typical scintillation noise in TORTORA observations.

$P(r)$ is the probability of obtaining $r$ photoelectrons for $N$ photons given a quantum efficiency of $p$. The mean is $\mu=$ $N p$, and the variance is $\sigma^{2}=N p(1-p)$.

3.3. Scintillation Noise. Additional uncertainty arises from atmospheric transmission variability. Scintillation is a dimensionless measure of the flux variation of a source observed through a finite aperture due to fluctuations in the refractive index of the atmosphere. Atmospheric scintillation adds an effective constant error term to the photometry. This error can be estimated from [11] as

$$
c_{\text {scint }}=0.09 \frac{X^{7 / 4}}{D^{2 / 3} \sqrt{2 t_{\exp }}}\left(\frac{\lambda}{550}\right)^{-7 / 12} \exp \left(\frac{h}{8000}\right)
$$

where $D$ is the telescope diameter in centimeters, $X$ is the airmass, $h$ is the observatory altitude in meters, $t_{\exp }$ is the exposure time in seconds, $\lambda$ is the wavelength in nanometers, and $c_{\text {scint }}$ is the scintillation noise in magnitudes. The precise value of the exponent of the airmass $X$ depends on the wind direction; it is equal to 2 when observing in the same direction as the wind and to 1.5 when observing perpendicular to it. Young [12] was able to show that for moderate airmass, the scintillation standard error is proportional to the 1.75 power of airmass but becomes asymptotically proportional to the $3 / 2$ power for very large airmass.

The $D^{-2 / 3}$ dependence may be applied only to exposures long enough to ignore all high-frequency content. Ryan and Sandler [13] have derived a $D^{-1.1 \pm 0.1}$ dependence for exposure times $\leq 0.25$ seconds, which agrees with Ellison and Seddon's [14] experimental data and Reiger's theoretical equations [15]. We adopt this diameter dependence in (2) to describe the scintillation noise in TORTORA photometry.
Taking into account the altitude of ESO Observatory $(h=2400 \mathrm{~m})$ and the aperture diameter of TORTORA telescope $(D=12 \mathrm{~cm})$, the estimated amount of scintillation is $0.01 \lesssim c_{\text {scint }} \lesssim 0.03$ magnitudes for moderate sky conditions with an airmass value $X$ between 1 and 2 in 0.13seconds photometric measurements (Figure 2).

\section{Photometry of the TORTORA Fields}

Circular aperture photometry of the TORTORA images was performed with PHOT/DAOPHOT function in IRAF (IRAF: Image Reduction and Analysis Facility; http://iraf .noao.edu/). Typically, the optimal radius of aperture was chosen to be 5 pixels for bright sources after the experiments with growth curves. Determining an appropriate aperture size to measure a total instrumental magnitude can be done by building up a light growth curve of the object. The light growth curve is the object's flux as a function of aperture radius. The adopted aperture would then reflect the point in the light growth curve where it flattens out. This optimal aperture size, which produces the smallest photometric error, contains also the spatially varying PSF due to geometric distortions present in the final images. A temporal sequence of contiguous light growth curves for as well as the values of the ellipticity and the Full Width at Half Maximum (FWHM) of the best fit Moffat profile is displayed in Figure 3. The growth curves were calculated selecting bright and isolated stars in different location of the widefield image. The images were acquired with a continuous exposures time of 0.13 seconds per frame under moderate sky condition. The values of the affective air mass and the seeing are estimated to be $\sim 1.30$ and $\sim 0.8^{\prime \prime}$, respectively (http://www.ls.eso.org/lasilla/dimm/). At the corresponding time the percent of the Moon illuminated is $6 \%$ at a distance 


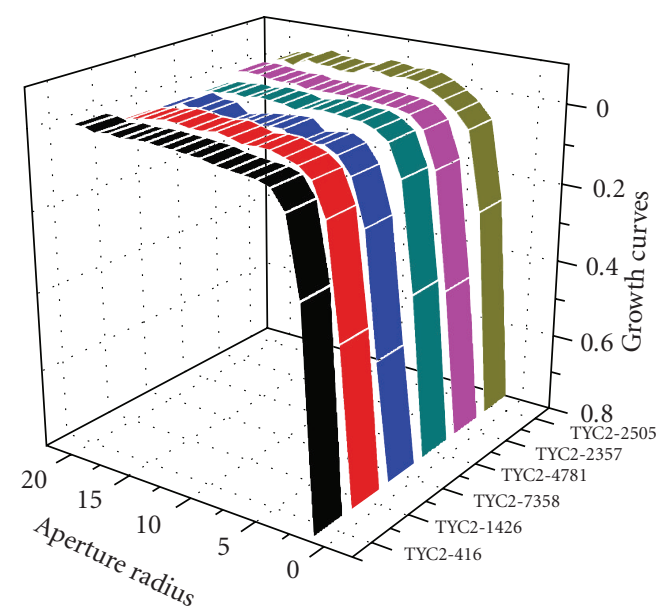

(a)

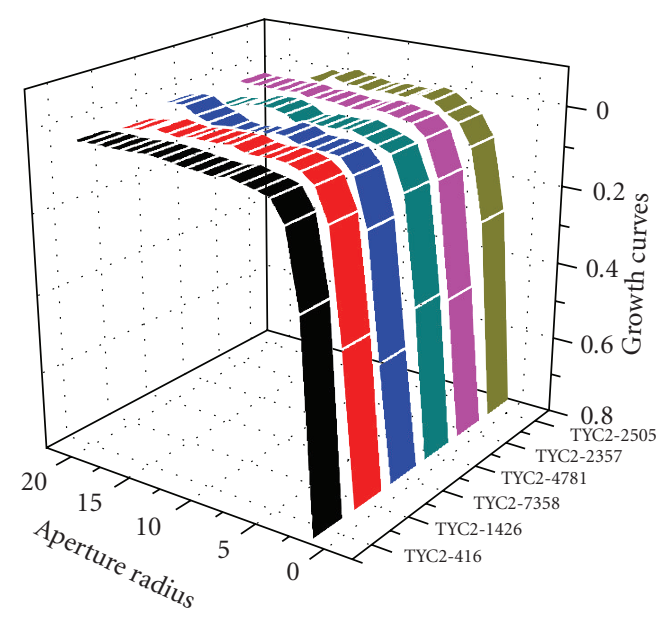

(c)

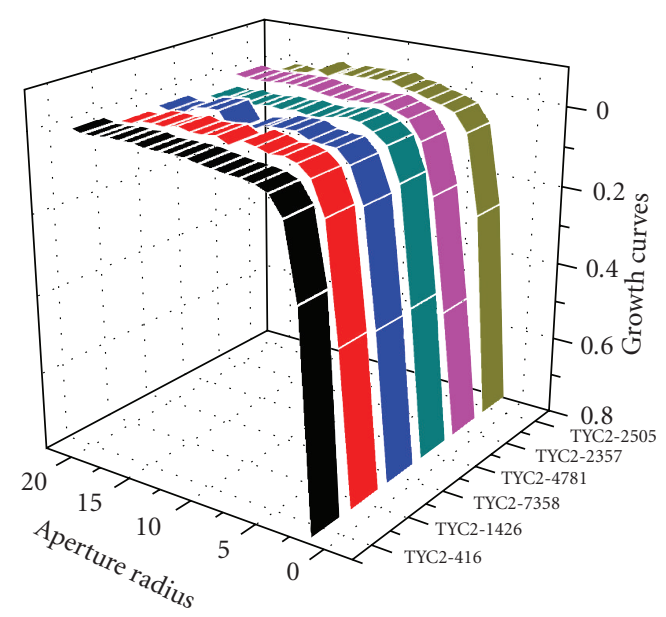

(e)
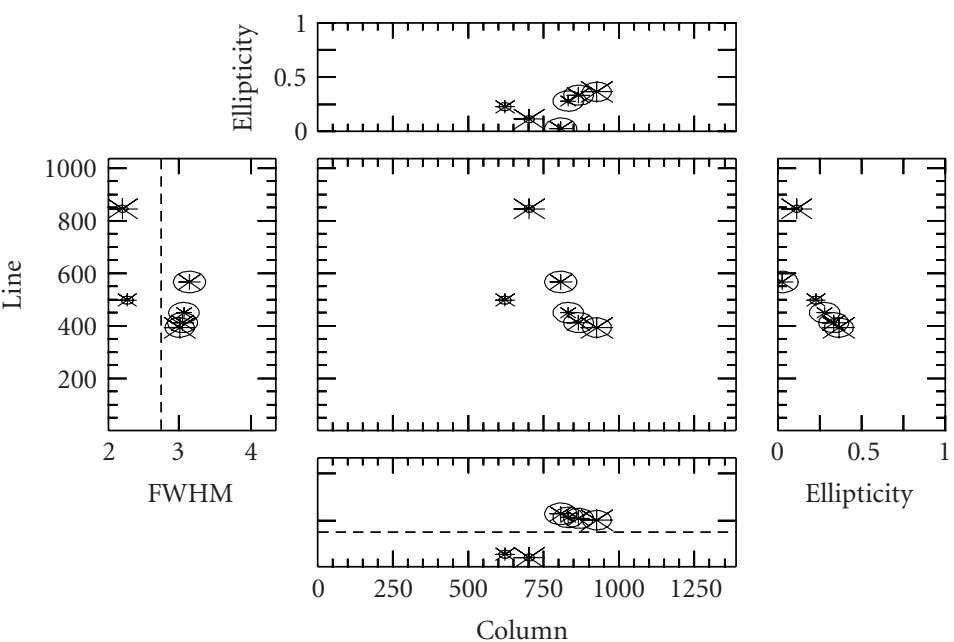

(b)
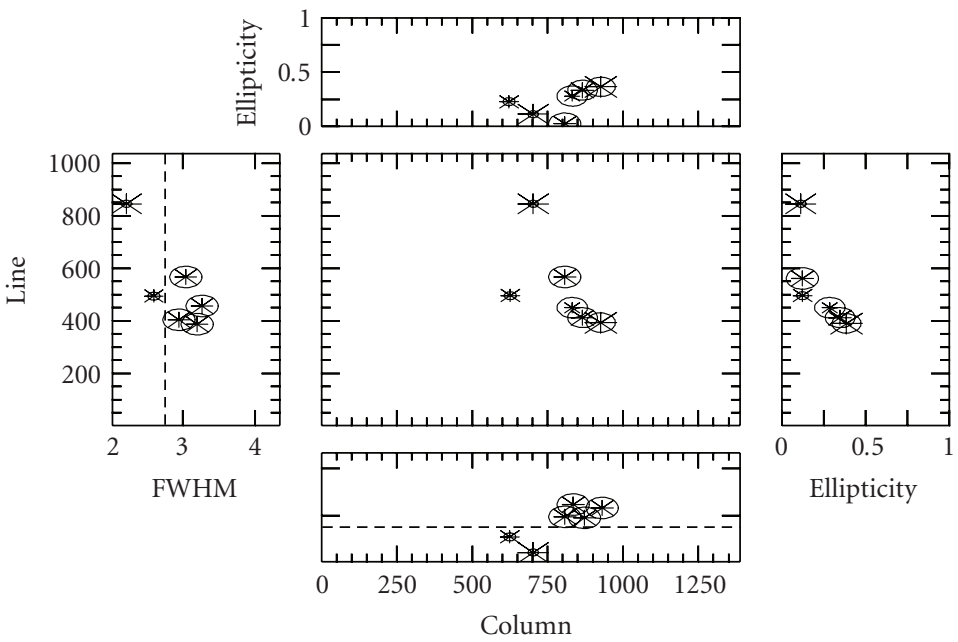

(d)
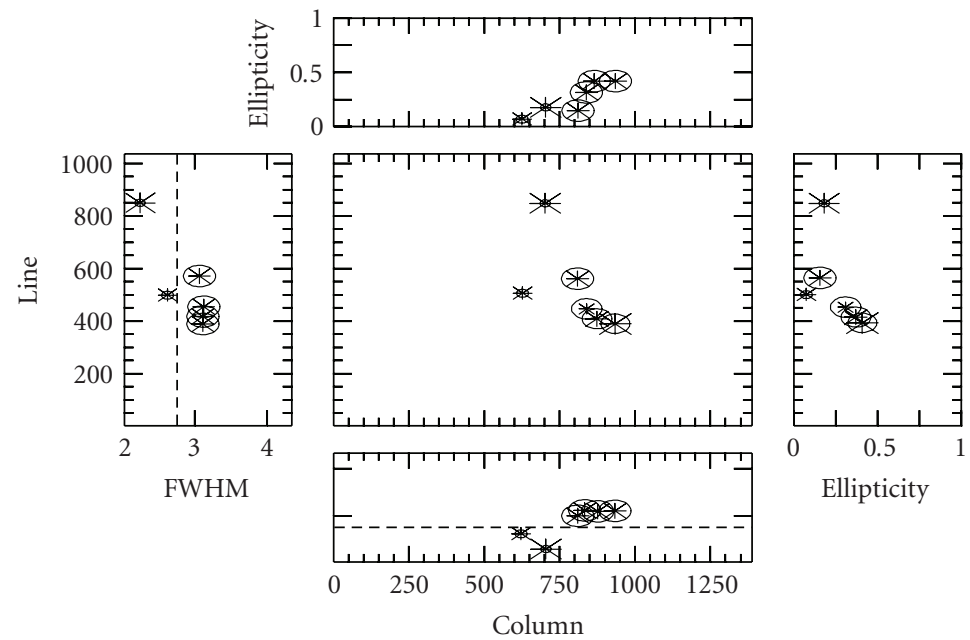

(f)

FIgURe 3: Continued. 


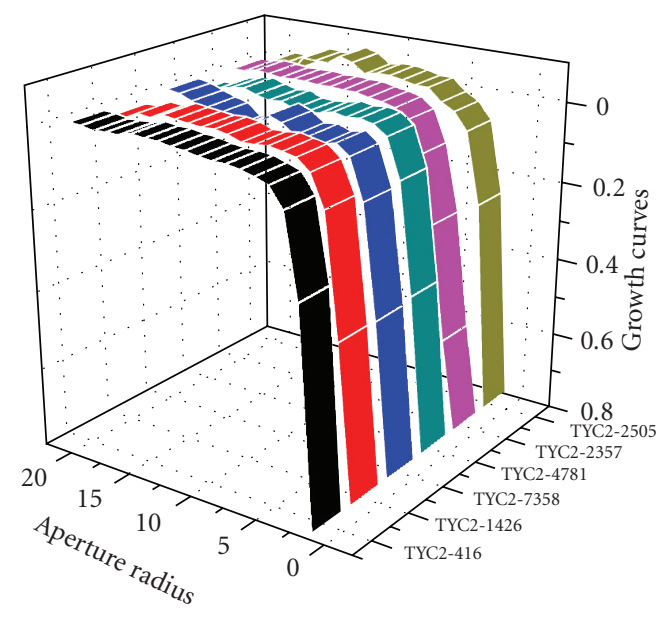

(f)

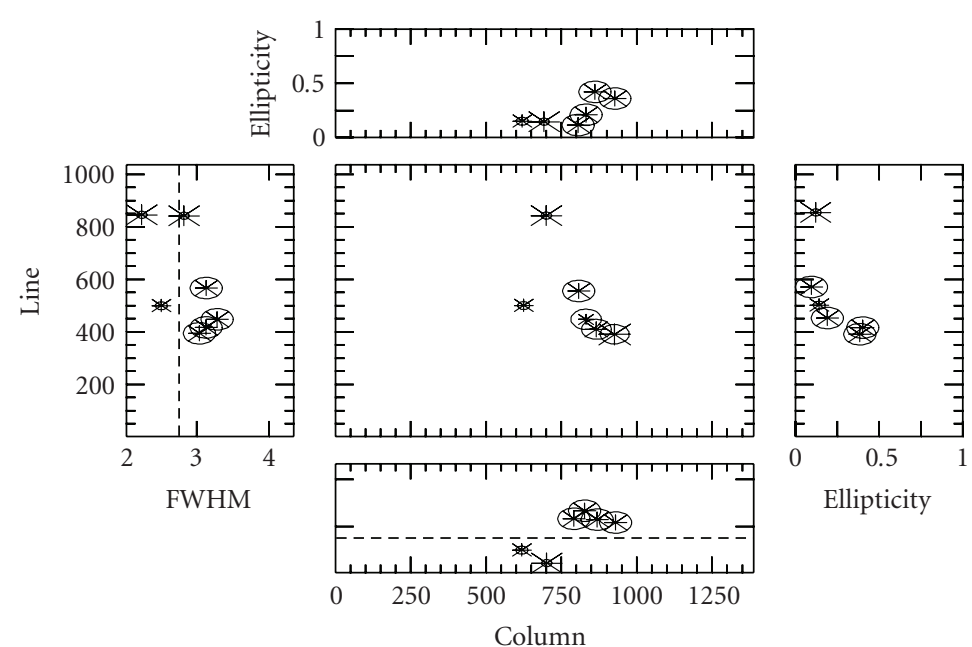

(g)

FIgURE 3: Example of growth curves acquired with a continuous exposure time of 0.13 seconds per frame, under moderate sky condition (see values in the text). The position of the stars may be found in the sky field of Figure 1 reading the the Tycho- 2 identification number (id number) labeled in $y$-axis. For each star are reported the values of the ellipticity, the FWHM using as best fit a Moffat profile, and the pixel coordinates.

of $126^{\circ}$ from the target. The position of the star may be found in the sky field displayed in Figure 1 reading the Tycho-2 identification number (id number) labeled in $y$-axis. The total flux of the stars is contained within well-determined radius showing a good homogeneity of image intensifier and CCD response. The asymptotic value is immediately apparent and constant with a satisfactory degree of accuracy. The short sequence collected here shows that the stability of the TORTORA system is enough to assure the reliability of the detected short-lived periodicity in the ultra-fast optical photometry.

The correction for the finite size of the aperture is called the aperture correction. It is defined simply as the magnitude difference between the asymptotic magnitude and the magnitude at the given aperture. Corrections for the amount of missing flux from the source outside the optimal aperture size were done using a correction factor estimated from the method proposed by Stetson [16] and implemented under IRAF task mkapfile. The aperture correction is typically of order $0.10 \mathrm{mag}$ at the center of the image $\left(10^{\circ} \times 10^{\circ}\right)$, while it reaches a maximum value of 0.20 mag at the edges of the frame in which the field has less sharpness due to the vignetting. Proper correction factor is systematically added to our photometry. Finally, by the use of very large circular apertures we test that extra-scattered light contribution around the source is so low that it can be neglected.

Sufficiently accurate results are also obtained under relatively suboptimal sky conditions. Figure 4 shows a contiguous sequence of standard stars detected within the limits of observability permitted by the instrument before the automatic protection shutdown. The images were acquired with a continuous exposures time of 0.13 seconds per frame. The plots combine an image, a surface plot of the image data and a contour plot of the images data in a single tri-level display. The $x$-axis and $y$-axis indicate the pixel coordinates place in the center of the FOV, and the $z$-axis represents the signal intensity. The values of the affective air mass and the seeing are estimated to be $\sim 2.61$ and $\sim 0.9^{\prime \prime}$, respectively. At the corresponding time the percent of the Moon illuminated is 98 at a distance of $58^{\circ}$ from the target. We emphasized that the autofocusing system is stable, and the stars retain their original position without emerging distortion effects.

4.1. Linearity and Dynamic Range. The spectral response of the system is primarily determined by the photocathode type used in the image intensifier. TORTORA was developed for ground optical observation, and a multialkali (S20) photocathode is employed. Its typical spectral response peaks near $440 \mathrm{~nm}$ (Figure 5). No photometric filter has been used with it.

By comparing the instrumental magnitudes with the standard stars tabulated in Tycho-2 catalogue (http://archive.eso.org/ASTROM/), we may test the linearity and the fractional coincidence loss of the instrument for a wide range of fluxes. Figure 6 shows the dependency of the measured instrumental magnitude and the standard $\mathrm{V}$ magnitude. This result spotlights a good linearity of the instrument. Furthermore, the fractional coincidence loss caused by dead time is negligible for the fluxes under consideration in TORTORA system. The stars with color index $(B-V)>1$ and $(B-V)<1$ are plotted with square and circle symbols, respectively. The reference Tycho-2 stars used to check the linear response of the camera are labeled in Figure 1. The values of the linear best-fits for the two samples (see the upper right box in Figure 6) are statistically comparable due to the large color term across the passband. Finally, using the linear fit equations reported in Figure 6, the photometry performed in instrumental system was converted to the V magnitudes of the Tycho-2 star catalogue. 


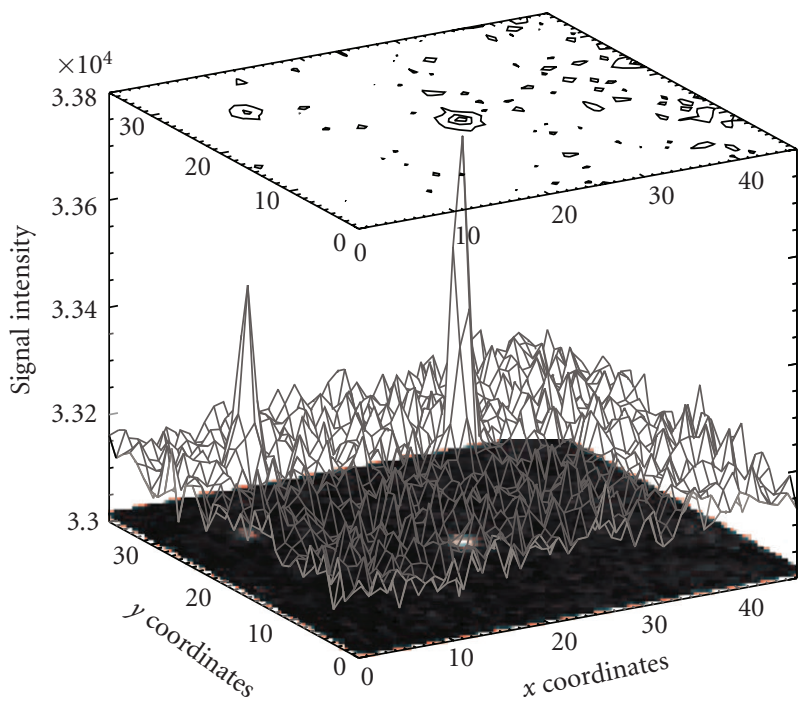

(a)

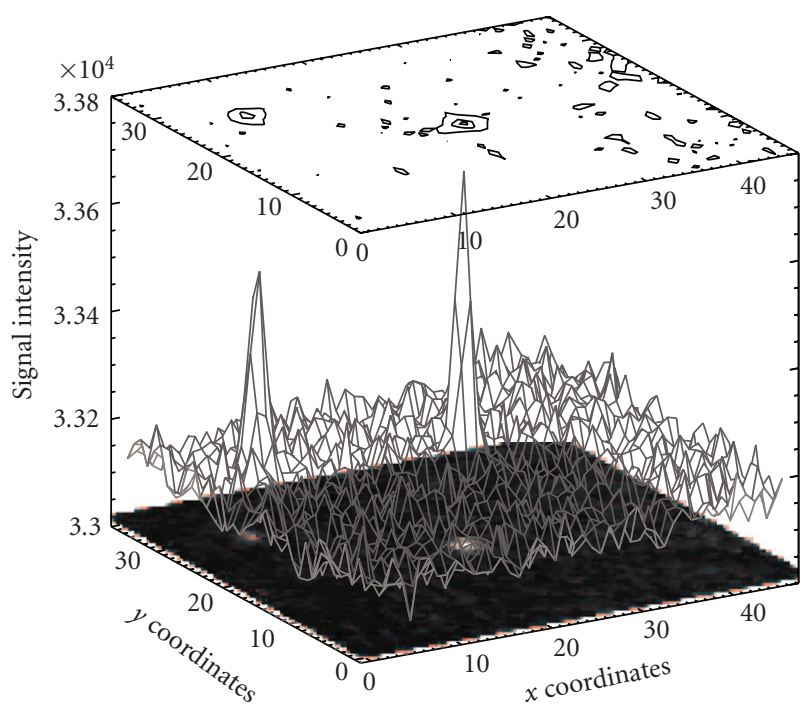

(c)

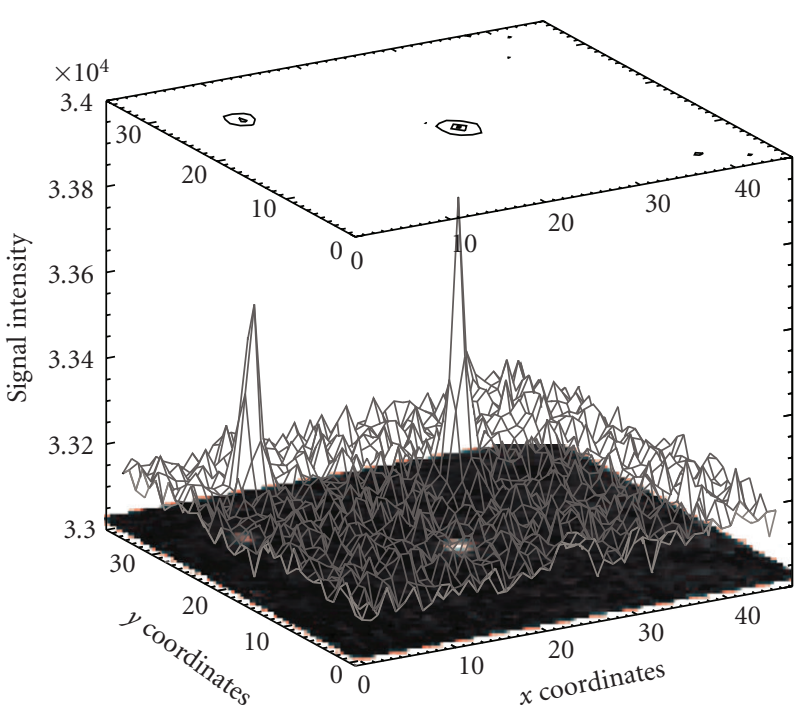

(b)

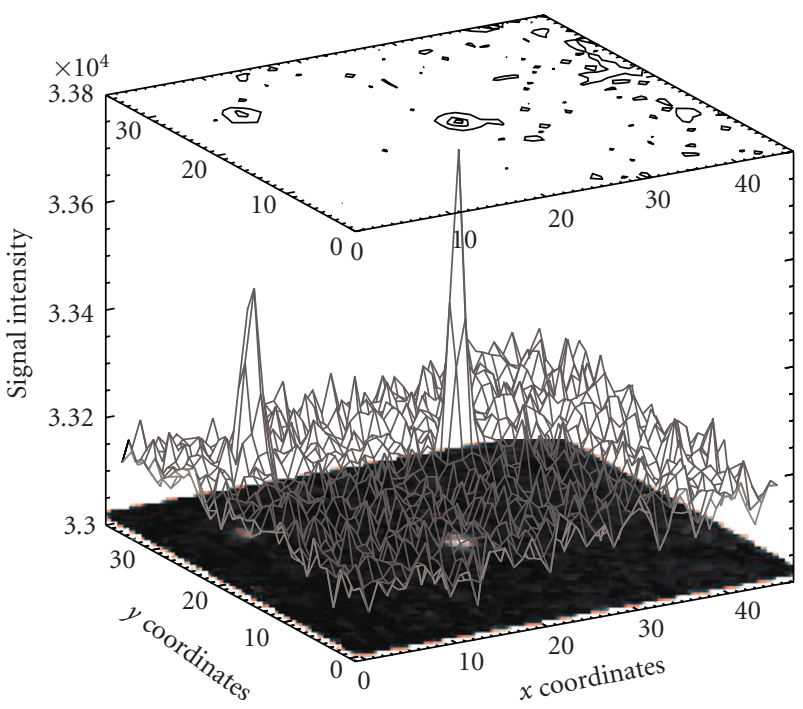

(d)

Figure 4: An example of observations acquired with continuous exposures time of 0.13 seconds per frame, under relative extreme sky conditions. The plots combine an image (image size: $2.5^{\circ} \times 2.5^{\circ}$ ), a surface plot of the image data, and a contour plot of the images data in a single tri-level display. The $x$-axis and $y$-axis indicate the pixel coordinates place in the center of the image frame at the position: RA(J2000) $=14 \mathrm{~h}: 31 \mathrm{~m}: 40.7 \mathrm{~s}, \mathrm{DEC}(\mathrm{J} 2000)=36 \mathrm{~d}: 18^{\prime}: 14.7^{\prime \prime}$ and the $z$-axis represents the signal intensity. The values of the affective air mass and the seeing are estimated to be $\sim 2.61$ and $\sim 0.9^{\prime \prime}$, respectively. At the corresponding time the percent of the Moon illuminated is $98 \%$ at a distance of $58^{\circ}$ from the target.

The calibration of the TORTORA instrumental magnitude to the visual band is preferable than the blue filter because the empirical correlation has a lower degree of dispersion and the dependence of color index is negligible.

\section{Discussion and Conclusion}

The photometric accuracy of the wide sky fields observed by the high speed TORTORA CCD camera equipped with an image intensifier is analyzed. The typical potential sources of instrumental artifacts such as (i) the spatial deformation of the image produced by the electron-optical input system (after electrons are ejected from the photocathode, they are collected and focused by the electric field of an electrodes configuration), (ii) blurring introduced by imperfections in the transmission optics between the phosphor screen and CCD, (iii) broadening of the light pulse produced by a fraction of photons emitted by the phosphor screen that travel parallel to the surface and scatter, (iv) stray light that arrives to the camera through multiple reflections from various detector components in the optical interface are negligible and do not affect the photometric results as expected from the previous laboratory measurements. Long 


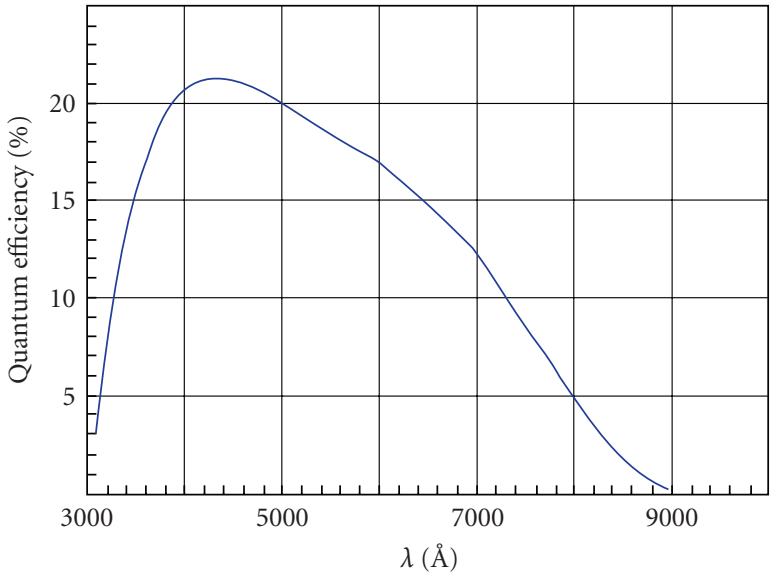

FIGURE 5: Spectral sensitivity curve of the multialkali (S20) photocathode employed in TORTORA.

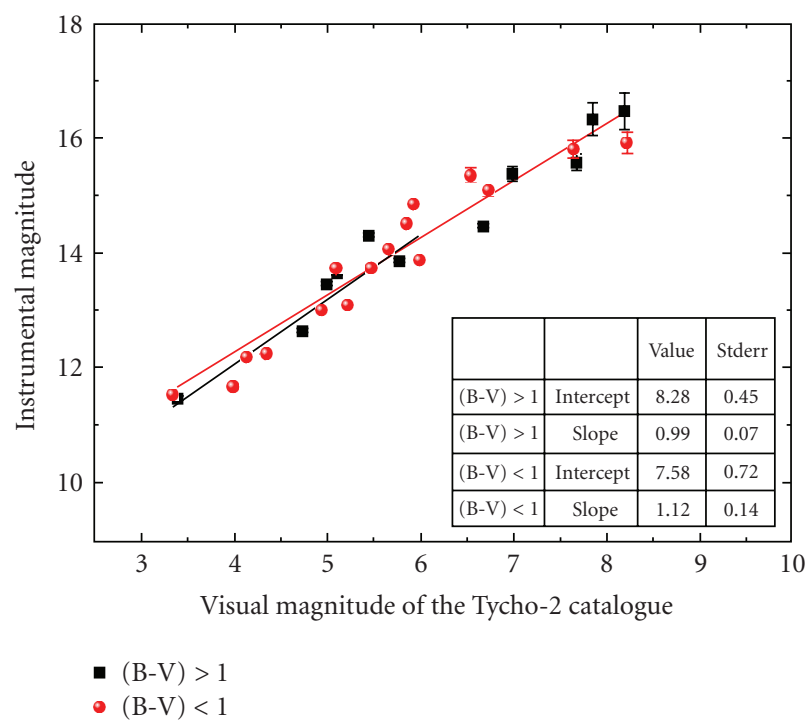

Figure 6: Instrumental magnitude versus standard Visual magnitude. The stars with color index $(\mathrm{B}-\mathrm{V})>1$ and $(\mathrm{B}-\mathrm{V})<1$ are plotted with square and circle symbols, respectively. The reference stars under consideration are labeled in Figure 1 using the Tycho-2 identification number (id number). The values of the linear best-fits are reported in the right upper box.

sequences of growth curves of bright and isolated standard stars show that the flux is contained within a well-determined and constant radius. Sequences of surface plot indicate that $(x, y)$ location of the event is preserved without appreciable degradation of its intensity profile. The example given here shows the performance of the TORTORA camera within the limits of observability permitted by the instrument before the automatic protection shutdown. However proper extra-Poissonian fluctuations caused by statistical variations produced by gain variation across the image intensifier have to be considered for the reliability of the detected short-lived periodicity (Section 3.2).
Since May 2006 we accumulated approximately 200 nights of observations. The fields of the GRB 060719 [17], GRB 061202 [18], and GRB 061218 [19] were observed 59 seconds, 92 seconds, and 118 seconds after the satellite trigger, respectively. Fourier analysis was performed to search for upper limit for periodic signal at the GRB position. In the remarkable case of the GRB 080319B, TORTORA began taking data 26 minutes before the satellite trigger and observed the optical flux coincident with the $\gamma$-ray emission with unprecedented level of accuracy, collecting $\sim 13320$ images. Long and short time-scale variability was detected during the naked-eye prompt emission [20].

One of the important results of our routine observation is the estimation of the detection rate of the short-lived orphan transients (in preparation). Currently the prompt optical transient detection rate determined by observations of TORTORA is less than $8 \times 10^{-9} \mathrm{deg}^{-2} \mathrm{sec}^{-1}$ for transients that are brighter than $10.5^{m}$ on 0.13 seconds timescale.

\section{Acknowledgments}

This work was supported by the University of Bologna Progetti Pluriennali 2003, by grants of CRDF (no. RP1-2394MO-02), RFBR (no. 04-02-17555, 06-02-08313, and 09-0212053), INTAS (04-78-7366), and by the Presidium of the Russian Academy of Sciences Program. The authors thank the referee for his/her diligent work and for providing several suggestions that have helped to clarify the results.

\section{References}

[1] A. Piccioni, C. Bartolini, A. Guarnieri, G. Pizzichini, J. M. Poulsen, and S. R. Rosellini, "A fast imaging photometer and the detection of optical flashes from gamma-ray burst sources," in Vulcano Workshop 1988: Frontier Objects in Astrophysics and Particle Physics, pp. 217-224, 1989.

[2] A. Piccioni, C. Bartolini, A. Guarnieri, and G. Pizzichini, "FIP_a fast imaging photometer for optical flash observations," in Gamma-Ray Bursts-Observations, Analyses and Theories (A93-20206 06-90), pp. 148-154, 1992.

[3] A. Piccioni, C. Bartolini, F. Cortecchia, G. Cosentino, A. Guarnieri, and G. Pizzichini, "FIP: a fast imaging photometer for the optical study of gamma ray bursts," Memorie della Societa Astronomia Italiana, vol. 67, pp. 569-575, 1996.

[4] G. M. Beskin, V. Plokhotnichenko, C. Bartolini, et al., "Catching the light curve of flaring GRBs: the opportunity offered by scanning telescopes," Astronomy and Astrophysics Supplement Series, vol. 138, no. 3, pp. 589-590, 1999.

[5] G. Beskin, C. Bartolini, A. Guarnieri, et al., "Simultaneous detection of the high energy and optical transients by Čerenkov telescopes," in Gamma-Ray Bursts in the Afterglow Era: Proceedings of the International Workshop, E. Costa, F. Frontera, and J. Hjorth, Eds., ESO Astrophysics Symposia, 2001, pp. 387-389, Springer, Rome, Italy, October 2000.

[6] A. Piccioni, C. Bartolini, A. Guarnieri, and G. Beskin, "Search for optical high energy transients by large light collectors," in Presented at the IHP-Users Workshop, 2001.

[7] S. Karpov, G. Beskin, A. Biryukov, et al., "Optical camera with high temporal resolution to search for transients in the wide field," Nuovo Cimento della Societa Italiana di Fisica C, vol. 28, no. 4-5, pp. 747-750, 2005. 
[8] E. Molinari, S. Bondar, S. Karpov, et al., "TORTOREM: twotelescope complex for detection and investigation of optical transients," Nuovo Cimento della Societa Italiana di Fisica B, vol. 121, no. 12, pp. 1525-1526, 2006.

[9] A. Biryukov, G. Beskin, S. Bondar, et al., "Software for detection of optical transients in observations with rapid wide-field camera," Astronomische Nachrichten, vol. 325, no. 6-8, p. 676, 2004.

[10] S. Karpov, G. Beskin, S. Bondar, et al., "Wide and fast. Monitoring the sky in sub-second domain," in Proceedings of Workshop on Robotic Autonomous Observatories, Malaga, Spain, May 2009.

[11] D. S. Birney, G. Gonzalez, and D. Oesper, Book-Review of Observational Astronomy, Cambridge University Press, Cambridge, UK, vol. 101, 2nd edition, 1991.

[12] A. T. Young, "Observational technique and data reduction," in Methods of Experimental Physics, N. Carleton, Ed., pp. 123$192,1974$.

[13] P. Ryan and D. Sandler, "Scintillation reduction method for photometric measurements," Publications of the Astronomical Society of the Pacific, vol. 110, no. 752, pp. 1235-1248, 1998.

[14] M. A. Ellison and H. Seddon, "Some experiments on the scintillation of stars and planets," Monthly Notices of the Royal Astronomical Society, vol. 112, pp. 73-87, 1952.

[15] S. H. Reiger, "Starlight scintillation and atmospheric turbulence," Astronomical Journal, vol. 68, pp. 395-406, 1963.

[16] P. B. Stetson, "On the growth-curve method for calibrating stellar photometry with CCDs," Publications of the Astronomical Society of the Pacific, vol. 102, pp. 932-948, 1990.

[17] A. Guarnieri, C. Bartolini, G. Beskin, et al., "GRB 060719: TORTOREM optical upper limits," GRB Coordinates Network, Circular Service, 5372, 1, 2006.

[18] S. Karpov, G. Beskin, S. Bondar, et al., "GRB 061202: TORTOREM optical upper limits," GRB Coordinates Network, Circular Service, 5897, 1, 2006.

[19] S. Karpov, G. Beskin, S. Bondar, et al., "GRB 061218: TORTOREM optical upper limits," GRB Coordinates Network, Circular Service, 5941, 1, 2006.

[20] G. Beskin, S. Karpov, S. Bondar, et al., "Fast optical variability of Naked-Eye Burst-manifestation of periodic activity of internal engine," http://arxiv.org/abs/0905.4431. 

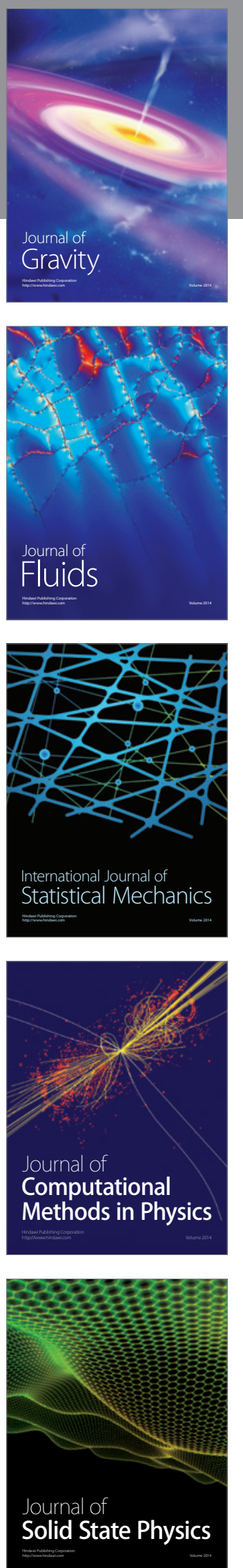

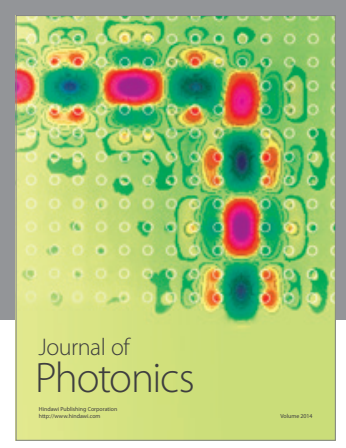

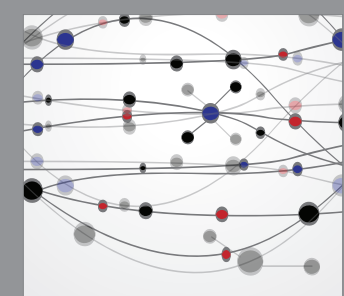

The Scientific World Journal
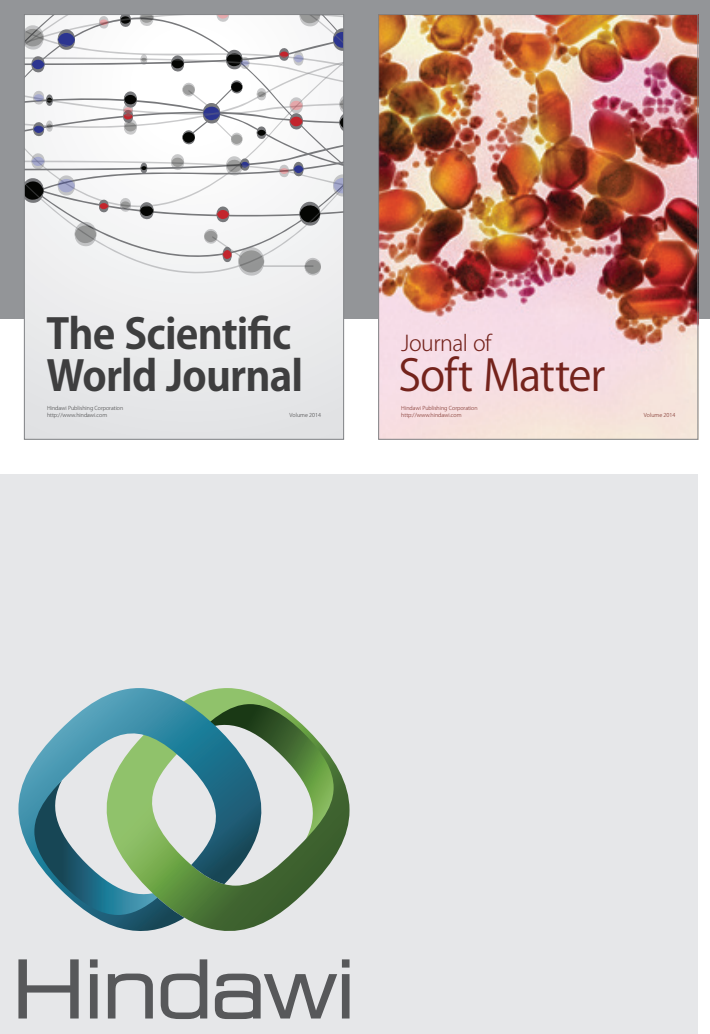

Submit your manuscripts at

http://www.hindawi.com
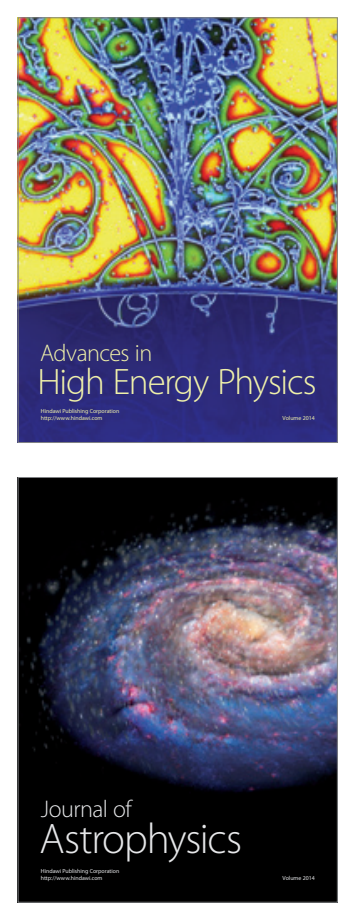
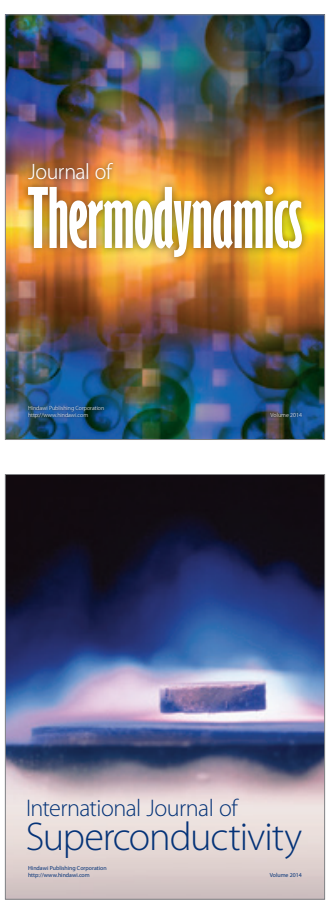
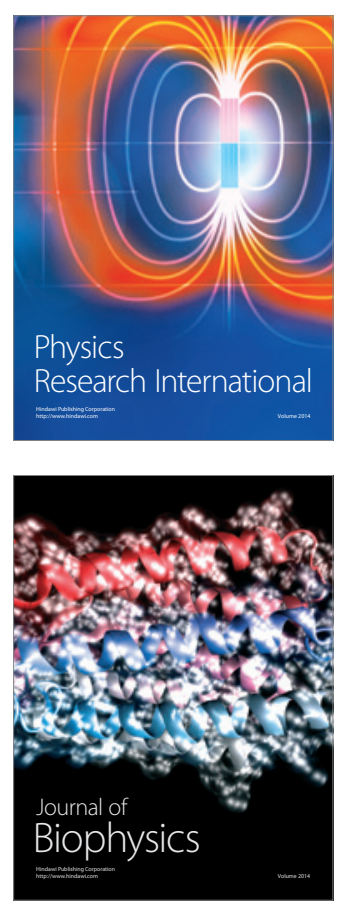
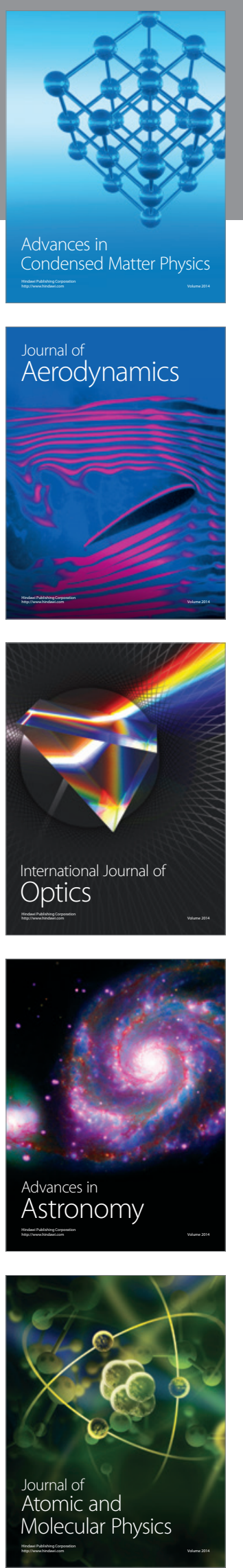